\title{
Effect of turbulence on zonal jet flows in equivalent-barotropic quasi-geostrophic model
}

\author{
K. Obuse $^{1} \quad$ S. Takehiro ${ }^{2} \quad$ M. Yamada ${ }^{3}$
}

Received 5 February 2017, revised 12 December 2017

\begin{abstract}
The linear stability of zonal flows superposed upon a sinusoidal, slightly supercritical background flow in an equivalent-barotropic quasigeostrophic model on a $\beta$ plane is investigated using asymptotic analysis. Consistent with results of full numerical simulations, all the steady isolated zonal jet solutions were found to be linearly unstable, and specifically, the mode independent of longitudinal direction was found to be most unstable, even when the amplitude equation of the zonal flows has longitudinal dependence.
\end{abstract}

DOI:10.21914/anziamj.v58i0.11782, (c) Austral. Mathematical Soc. 2017. Published 2017-12-14, as part of the Proceedings of the 18th Biennial Computational Techniques and Applications Conference. ISSN 1445-8810. (Print two pages per sheet of paper.) Copies of this article must not be made otherwise available on the internet; instead link directly to the DOI for this article. 


\section{Contents}

1 Introduction

C176

2 Amplitude equation and steady solution

C177

3 Linear stability of steady solution

C181

$3.1 \xi$-independent case . . . . . . . . . . . . . . . C C183

$3.2 \xi$-dependent case . . . . . . . . . . . . . . . . . C184

4 Conclusions

C186

References

C187

\section{Introduction}

It is well known that in forced two-dimensional turbulence on a rotating sphere, a multiple zonal-band structure, i.e., a structure with alternating eastward and westward jets, develops in time [1,2]. Then, for example, in the case of the two-dimensional barotropic model, the multiple zonalband structure experiences intermittent mergers and disappearances of zonal jets [1]. With the view to understanding such behaviour of zonal flows in twodimensional barotropic turbulence in rotating systems, Manfroi and Young [3] considered large-scale zonal flow superposed upon a homogeneous zonal flow and a small-scale sinusoidal transverse flow on a $\beta$ plane, and derived a longitude-independent amplitude equation for zonal flows. Obuse et al. [4] then obtained analytical steady isolated zonal jet solutions of Manfroi and Young's model, and investigated their linear stability. They found that the instability of the zonal jet solutions and the temporal development of the instability are consistent with the disappearance of zonal jets seen in full numerical simulations of the two-dimensional barotropic model on rotating spheres. This suggests that although Manfroi and Young's equation is a highly 
simplified model of a zonal flow under the influence of zonal flow-turbulence interaction, it captures many fundamental dynamics of zonal flows in twodimensional turbulence on a rotating sphere and a $\beta$ plane. Hence, in this paper we consider the equivalent-barotropic quasi-geostrophic model, where the surface variation of the fluid layer is included in the two-dimensional barotropic system. Then we extend Manfroi and Young's model, to investigate the linear stability of zonal jets.

\section{Amplitude equation and steady solution}

We consider zonal flows superposed upon a small-scale sinusoidal transverse flow in an equivalent-barotropic quasi-geostrophic model, and follow the asymptotic analysis of Manfroi and Young [3], to derive an amplitude equation for zonal flows.

In the derivation of the amplitude equation of zonal flows [3, equation (4.1)], Manfroi and Young only considered a longitude-independent leading order stream function perturbation. In reality, zonal jets in the forced equivalentbarotropic quasi-geostrophic model are governed by two-dimensional equations. Therefore, we consider when the stream function is longitude-dependent.

The dynamics of incompressible flow in the forced equivalent-barotropic quasi-geostrophic model are described by the vorticity equation

$$
\mathrm{Z}_{\mathrm{t}}+\mathrm{J}(\Psi, \mathrm{Z})+\beta \Psi_{x}-\frac{1}{\mathrm{~L}_{\mathrm{d}}^{2}} \Psi_{\mathrm{t}}=\mathrm{F}+\nu \nabla^{2} \mathrm{Z}
$$

Here $t$ is the time, $\Psi$ is the stream function, $Z=\nabla^{2} \Psi$ is the vorticity, $F$ is the vorticity forcing function, $v$ is the kinematic viscosity coefficient, and $\beta$ is the beta parameter. We have also introduced the Rossby radius of deformation $\mathrm{L}_{\mathrm{d}}=\mathrm{gH} / \mathrm{f}_{0}^{2}$, where $\mathrm{g}$ is the magnitude of gravitational acceleration, $\mathrm{H}$ is the width of the zonal channel, and $f_{0}$ is the Coriolis parameter at the reference latitude, respectively. The Jacobian operator is defined as $J(A, B)=$ $(\partial A / \partial x)(\partial B / \partial y)-(\partial A / \partial y)(\partial B / \partial x)$, and $\nabla=(\partial / \partial x, \partial / \partial y)$. 
We consider the same situation as Manfroi and Young [3], i.e., a steady sinusoidal base flow with the velocity

$$
\left(u_{B}, v_{B}\right)=\left(-\frac{\partial \Psi_{B}}{\partial y}, \frac{\partial \Psi_{B}}{\partial x}\right)=\left(u_{B}, m \Psi_{B 0} \sin m x\right)
$$

which is driven by a suitable forcing function, where $\Psi_{\mathrm{B}}, \mathfrak{u}_{\mathrm{B}}$ and $\nu_{\mathrm{B}}$ are the stream function, $x$ and $y$ velocity components of the base flow, respectively. The parameters $\mathrm{U}_{\mathrm{B}}$ and $\Psi_{\mathrm{B} 0}$ are constant, and $m$ is an integer. We also assume the Reynolds number of the base flow to be $R=\Psi_{B 0} / v=R_{c}\left(1+\epsilon^{2}\right)$. Here, $R_{c}$ is the critical Reynolds number, and $\epsilon$ is a small quantity. Then we write the total stream function of the flow as $\Psi=\Psi_{B}(x, y)+\psi(x, y, t)$, where $\psi(x, y, t)$ is the perturbation stream function.

We first introduce dimensionless variables defined as

$$
(\hat{x}, \hat{y})=(m x, m y), \quad \hat{t}=t^{2} v, \quad \hat{\psi}=\frac{\psi}{v}, \quad \hat{u}_{B}=\frac{u_{B}}{m v}, \quad \hat{\beta}=\frac{\beta}{m^{3} v},
$$

then the stream function of the disturbance flow satisfies a non-dimensionalised equation

$$
\frac{\partial \hat{\zeta}}{\partial \hat{t}}+\hat{u}_{B} \frac{\partial \hat{\zeta}}{\partial \hat{x}}+R\left(\frac{\partial \hat{\zeta}}{\partial \hat{y}}+\frac{\partial \hat{\psi}}{\partial \hat{y}}\right) \sin \hat{x}+J(\hat{\psi}, \hat{\zeta})+\hat{\beta} \frac{\partial \hat{\psi}}{\partial \hat{x}}-\frac{1}{L_{d}^{2}} \frac{\partial \hat{\psi}}{\partial \hat{t}}=\hat{\nabla}^{2} \hat{\zeta}
$$

where $\hat{\zeta}=\hat{\nabla}^{2} \hat{\psi}$ is the vorticity of the disturbance flow. Hereafter, we drop the notation ${ }^{\wedge}$ for the dimensionless variables and operators for simplicity.

Following Manfroi and Young [3], we then define ${ }^{1}$

$$
\eta=\epsilon y, \quad \tau=\epsilon^{4} t, \quad \xi=\epsilon^{6} x .
$$

${ }^{1}$ The scaling of $\eta$ and $\tau$ are chosen from the discussion for the band width of unstable wavenumbers and the growth rate of the instability of sinusoidal shear flow considered as a part of background flow, respectively. The scaling of $\xi$ is chosen so that the maximum number of physical processes appear in the final amplitude equation for the perturbation [3]. 
Now we perform a perturbation expansion

$$
\begin{cases}\psi=\psi_{0}+\epsilon \psi_{1}+\epsilon^{2} \psi_{2}+\cdots, & \mathrm{U}_{\mathrm{B}}=\mathrm{U}_{\mathrm{B} 0}+\epsilon \mathrm{U}_{\mathrm{B} 1}+\epsilon^{2} \mathrm{U}_{\mathrm{B} 2}+\cdots, \\ \beta=\beta_{0}+\epsilon \beta_{1}+\epsilon^{2} \beta_{2}+\cdots, & \frac{1}{\mathrm{~L}_{\mathrm{d}}}=\frac{1}{\mathrm{~L}_{0}}+\epsilon \frac{1}{\mathrm{~L}_{1}}+\cdots\end{cases}
$$

and multiple-scale expansion ${ }^{2}$

$$
\frac{\partial}{\partial x} \rightarrow \frac{\partial}{\partial x}+\epsilon^{6} \frac{\partial}{\partial \xi}, \quad \frac{\partial}{\partial y} \rightarrow \epsilon \frac{\partial}{\partial \eta}, \quad \frac{\partial}{\partial t} \rightarrow \epsilon^{4} \frac{\partial}{\partial \tau} .
$$

Substituting variables and operators (4), (5), and (6) into equation (3), and seeking an amplitude equation for the $\mathrm{O}(1)$ velocity element of the disturbance flow

$$
u(\xi, \eta, \tau)=-\frac{\partial \psi_{0}}{\partial \eta}
$$

we obtain

$$
\begin{array}{r}
\mathrm{u}_{\tau \eta \eta}-\left(1 / \mathrm{L}_{1}^{2}\right) \mathrm{U}_{\tau}=-\left(2-\gamma^{2}\right) \mathrm{U}_{\eta \eta \eta \eta}-3 \mathrm{U}_{\eta \eta \eta \eta \eta \eta}-2 \gamma\left(\mathrm{U}^{2}\right)_{\eta \eta \eta \eta} \\
+(2 / 3)\left(\mathrm{U}^{3}\right)_{\eta \eta \eta \eta}-\beta_{0} \mathrm{U}_{\xi},
\end{array}
$$

where

$$
\gamma=\beta_{1}-\mathrm{U}_{\mathrm{B} 1}
$$

Now, we consider a steady solution $\mathrm{U}_{0}(\eta)$ of equation (7), having one extrema and $\mathrm{U}_{0} \rightarrow \mathrm{U}_{W}$ as $\eta \rightarrow \pm \infty$, where $\mathrm{U}_{W}$ is a constant [4]. We first integrate (7) twice with respect to $\eta$, and use the conditions

$$
\mathrm{U}_{0} \rightarrow \mathrm{U}_{\mathrm{W}}, \quad \frac{\mathrm{du}_{0}}{\mathrm{~d} \eta} \rightarrow 0 \quad \text { as } \eta \rightarrow \pm \infty
$$

${ }^{2}$ Although the stream function of the disturbance flow $\psi$ has $\mathrm{O}(1)$ element $\psi_{0}$ in (5), the velocity of the disturbance flow is not $\mathrm{O}(1)$, since, by consindering an equation for $\psi_{0}$, it is confirmed that $\psi_{0}$ has no $x$-dependence [3, equations (27.a)-(28)]). 
Multiplying the resulting equation by $\mathrm{dU}_{0} / \mathrm{d} \eta$, and integrating again with respect to $\eta$, we obtain

$$
\frac{\mathrm{d} \mathrm{U}_{0}}{\mathrm{d \eta}}= \pm \frac{1}{3} \sqrt{-\mathrm{V}\left(\mathrm{U}_{0}\right)}
$$

Here, the potential is defined as

$$
\mathrm{V}\left(\mathrm{U}_{0}\right)=-\mathrm{U}_{0}^{4}+4 \gamma \mathrm{U}_{0}^{3}+3\left(2-\gamma^{2}\right) \mathrm{U}_{0}^{2}-6 \mathrm{C}_{1} \mathrm{U}_{0}-3 \mathrm{C}_{2},
$$

and the constants of integration $\mathrm{C}_{1}$ and $\mathrm{C}_{2}$ are

$$
\left\{\begin{array}{l}
\mathrm{c}_{1}=\left(2-\gamma^{2}\right) \mathrm{u}_{\mathrm{W}}+2 \gamma \mathrm{u}_{\mathrm{W}}^{2}-\frac{2}{3} \mathrm{u}_{\mathrm{W}}^{3} \\
\mathrm{C}_{2}=\mathrm{u}_{\mathrm{W}}^{4}-\frac{8}{3} \gamma \mathrm{u}_{\mathrm{W}}^{3}-\left(2-\gamma^{2}\right) \mathrm{u}_{W}^{2}
\end{array}\right.
$$

In order that the solution $U_{0}$ takes the same value $U_{W}$ at $\eta \rightarrow \pm \infty$, the potential $\mathrm{V}\left(\mathrm{U}_{0}\right)$ should have a double root $\mathrm{U}_{W}$ and two other distinct real roots $U_{E}$ and $U_{R}$, i.e.,

$$
\mathrm{V}\left(\mathrm{U}_{0}\right)=\left(\mathrm{U}_{0}-\mathrm{U}_{\mathrm{W}}\right)^{2}\left(\mathrm{U}_{0}-\mathrm{U}_{\mathrm{E}}\right)\left(\mathrm{U}_{0}-\mathrm{U}_{\mathrm{R}}\right),
$$

where $\mathrm{U}_{\mathrm{E}}<\mathrm{U}_{\mathrm{R}}, \mathrm{U}_{\mathrm{W}} \neq \mathrm{U}_{\mathrm{E}}, \mathrm{U}_{\mathrm{W}} \neq \mathrm{U}_{\mathrm{R}}$, and the conditions

$$
\gamma-\frac{1}{2} \sqrt{6\left(\gamma^{2}+2\right)}<\mathrm{U}_{W}<\gamma+\frac{1}{2} \sqrt{6\left(\gamma^{2}+2\right)},
$$

and

$$
\mathrm{U}_{\mathrm{W}}<\gamma-\frac{1}{2} \sqrt{2\left(\gamma^{2}+2\right)} \quad \text { or } \quad \gamma+\frac{1}{2} \sqrt{2\left(\gamma^{2}+2\right)}<\mathrm{U}_{\mathrm{W}}
$$

must be satisfied.

From (9), (10), and (11), we obtain

$$
\begin{aligned}
& \mathrm{U}_{\mathrm{E}}=2 \gamma-\mathrm{u}_{\mathrm{W}}-\sqrt{-2 \mathrm{u}_{W}^{2}+4 \gamma \mathrm{u}_{W}+\gamma^{2}+6}, \\
& \mathrm{U}_{\mathrm{R}}=2 \gamma-\mathrm{u}_{W}+\sqrt{-2 \mathrm{u}_{W}^{2}+4 \gamma \mathrm{u}_{W}+\gamma^{2}+6} .
\end{aligned}
$$


The steady isolated zonal jet solutions

$$
\mathrm{U}_{\text {eeast }}(\eta)=\frac{\mathrm{a}_{\text {east }}^{2} \mathrm{U}_{\mathrm{R}} \tanh ^{2}\left[\frac{\left(\mathrm{U}_{\mathrm{R}}-\mathrm{u}_{\mathrm{W}}\right) \mathrm{a}_{\text {east }}}{6} \eta\right]-\mathrm{U}_{\mathrm{E}}}{\mathrm{a}_{\text {east }}^{2} \tanh ^{2}\left[\frac{\left(\mathrm{U}_{\mathrm{R}}-\mathrm{u}_{W}\right) \mathrm{a}_{\text {east }}}{6} \eta\right]-1}, \mathrm{a}_{\text {east }}=\sqrt{\frac{\mathrm{U}_{\mathrm{E}}-\mathrm{U}_{W}}{\mathrm{U}_{\mathrm{R}}-\mathrm{U}_{W}}}
$$

and

$$
\mathrm{U}_{0 \text { west }}(\eta)=\frac{\mathrm{a}_{\text {west }}^{2} \mathrm{u}_{\mathrm{E}} \tanh ^{2}\left[\frac{\left(\mathrm{U}_{\mathrm{E}}-\mathrm{U}_{\mathrm{W}}\right) \mathrm{a}_{\text {west }}}{6} \eta\right]-\mathrm{U}_{\mathrm{R}}}{\mathrm{a}_{\text {west }}^{2} \tanh ^{2}\left[\frac{\left(\mathrm{u}_{\mathrm{E}}-\mathrm{U}_{\mathrm{W}}\right) \mathrm{a}_{\text {west }}}{6} \eta\right]-1}, \mathrm{a}_{\text {west }}=\sqrt{\frac{\mathrm{U}_{\mathrm{R}}-\mathrm{U}_{W}}{\mathrm{U}_{\mathrm{E}}-\mathrm{U}_{W}}},
$$

for parameters $\gamma$ and $\mathbf{U}_{W}$ satisfying conditions (12) and (13).

These solutions represents a zonal flow superposed upon a small-scale sinusoidal transverse background flow, governed by an amplitude equation having $\xi$ - and $\eta$ - dependence. Then $\boldsymbol{U}_{\xi}=0$ in equation (7) corresponds to the situation where $U$ is $\xi$-independent and governed by an amplitude equation depending only on $\eta$ and $\tau$.

\section{Linear stability of steady solution}

To investigate the linear stability of the steady solution $\mathrm{U}_{0}(\eta)$, we add a sufficiently small perturbation $v(\xi, \eta, \tau)=\exp (\sigma \tau) h(\xi) f(\eta)$. The characteristic equation is derived in the same manner as by Obuse et al. [4]. Substituting $\mathrm{U}_{0}+v$ into equation (7) and linearising with respect to $v$, then introducing a function $g(\eta)$, which satisfies $f(\eta)=d^{2} g(\eta) / d \eta^{2}$ and $g \rightarrow 0$, dg/d $\rightarrow 0$ as 
$\eta \rightarrow \pm \infty$, we obtain the characteristic equation

$$
\begin{aligned}
& \sigma\left[\frac{d^{2}}{d \eta^{2}}-\frac{1}{L_{1}^{2}}\right](g h) \\
= & -\left(2-\gamma^{2}\right) \frac{d^{4}(g h)}{d \eta^{4}}-3 \frac{d^{6}(g h)}{d \eta^{6}}+\frac{d^{2}}{d \eta^{2}}\left[\left(2 u_{0}^{2}-4 \gamma u_{0}\right) \frac{d^{2}(g h)}{d \eta^{2}}\right]-\beta_{0} g \frac{d h}{d \xi} .
\end{aligned}
$$

Since the $\xi$-dependence in equation (16) only appears in the form $\mathrm{dh}(\xi) / \mathrm{d} \xi$, by considering a Fourier expansion $h(\xi)=\sum_{n_{\xi}=-\infty}^{\infty} h_{n_{\xi}} \exp \left(i n_{\xi}\left(2 \pi / L_{\xi}\right) \xi\right)$, where $n_{\xi} \in \mathbb{N}$ is the wavenumber and $L_{\xi}$ is a width of the domain in the $\xi$ direction, it is sufficient to solve

$$
\begin{aligned}
& \sigma\left[\frac{d^{2}}{d \eta^{2}}-\frac{1}{L_{1}^{2}}\right] g \\
= & -\left(2-\gamma^{2}\right) \frac{d^{4} g}{d \eta^{4}}-3 \frac{d^{6} g}{d \eta^{6}}+\frac{d^{2}}{d \eta^{2}}\left[\left(2 u_{0}^{2}-4 \gamma u_{0}\right) \frac{d^{2} g}{d \eta^{2}}\right]-i \frac{2 \pi \eta_{\xi}}{L_{\xi}} \beta_{0} g(\eta),
\end{aligned}
$$

for various $2 \pi \beta_{0} n_{\xi} / L_{\xi}$. Because of the symmetry property of the characteristic equation discussed by Obuse et al. [4], investigating the linear stability of $\mathrm{U}_{0 \text { east }}$ with $\gamma \geqslant 0$ is sufficient to understand the linear stability for all $\mathrm{U}_{0}$. It is easily verified that the characteristic equation (17) also holds for $\sigma=0$.

We numerically solve the eigenvalue problem (17) by the Fourier spectral method, where, for example, $\mathrm{U}_{0}=\sum_{k=-k}^{k} \mathfrak{u}_{k} \exp \left(i k\left(2 \pi / L_{\eta}\right) \eta\right)$. We consider a periodic domain $\left[0, L_{\eta}\right]$. The width of the domain, $L_{\eta}$, is determined so that the numerical calculations converge. ${ }^{3}$ The size of the spatial grids is set to be $1 / 2^{10}$, and the truncation mode number $\mathrm{K}$ is chosen such that $\mathrm{K} / \mathrm{L}_{\eta}=125 / 16$ for each case. The $\mathrm{O}(\epsilon)$ element of the Rossby radius of deformation, $\mathrm{L}_{1}$, is always expressed by the ratio $\mathrm{L}_{1} / \mathrm{L}_{\mathrm{J}}$, where $\mathrm{L}_{\mathrm{J}}$ is the width of the steady zonal jet $\mathrm{U}_{0}$ and satisfies $\left|\left(\mathrm{U}_{0}(0)-\mathrm{U}_{0}\left(\mathrm{~L}_{\mathrm{J}} / 2\right)\right) /\left(\mathrm{U}_{0}(0)-\mathrm{U}_{W}\right)\right|=1 /$ e.

\footnotetext{
${ }^{3}$ We mainly took $\mathrm{L}_{\eta}=384$ and 512 for each case and confirmed the convergence of the calculations.
} 
Figure 1: Maximal growth rate of $(18)\left(\right.$ red crosses) for $\left(\gamma, \mathbf{U}_{W}\right)=(1.0,-1.0)$ (left) and $(5.0,-1.0)$ (right). The pink dotted line corresponds to the equivalent result for the barotropic model.
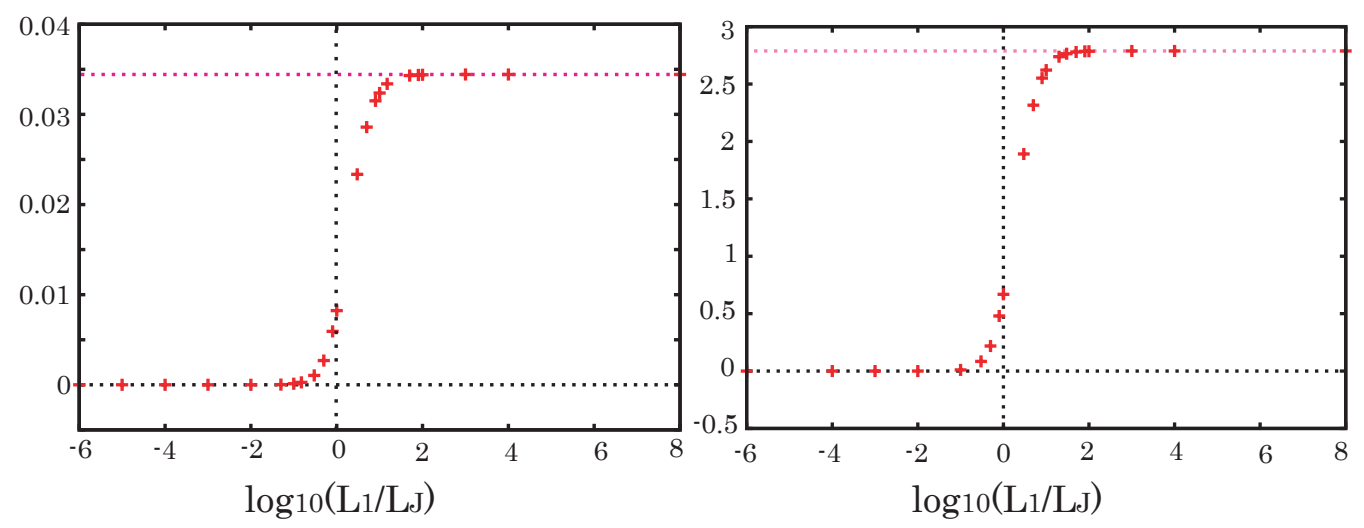

\section{$3.1 \xi$-independent case}

First, consider the case where $\mathrm{U}$ is independent of $\xi$. Thus the characteristic equation is

$$
\sigma\left[\frac{d^{2}}{d \eta^{2}}-\frac{1}{L_{1}^{2}}\right] g=-\left(2-\gamma^{2}\right) \frac{d^{4} g}{d \eta^{4}}-3 \frac{d^{6} g}{d \eta^{6}}+\frac{d^{2}}{d \eta^{2}}\left[\left(2 u_{0}^{2}-4 \gamma U_{0}\right) \frac{d^{2} g}{d \eta^{2}}\right] .
$$

The real parts of leading eigenvalues or maximal growth rate of perturbations are shown in Figure 1. When $\mathrm{L}_{1} / \mathrm{L}_{\mathrm{J}}$ is large, the maximal growth rate asymptote to those of $\mathrm{U}_{0}$ in a barotropic system obtained by Obuse et al. [4] (the pink dotted line in Figure 1). This is to be expected, since the vorticity equation of an equivalent-barotropic quasi-geostrophic model converges to a barotropic model when $\mathrm{L}_{d} \rightarrow \infty$. The maximal growth rate decreases as $\mathrm{L}_{1} / \mathrm{L}_{J}$ decreases, and appear to asymptote to zero. In this limit, varying $\mathrm{L}_{1} / \mathrm{L}_{\mathrm{J}}$ only corresponds to investigating the same instability on a different time scale. Therefore the real part of the leading eigenvalue is positive, not zero, even 
when $\mathrm{L}_{1} / \mathrm{L}_{\mathrm{J}}=1.0 \times 10^{-6}$. As a consequence, all the steady isolated jet solutions $\mathrm{U}_{0}$ are linearly unstable, as for the barotropic case of Obuse et al. [4], though the instability is weaker than for the barotropic case. The instability would be expected to bring about the deformation of $\mathbf{U}_{0}$, and cause the disappearance of zonal jets.

\section{$3.2 \xi$-dependent case}

Now we investigate the $\xi$-dependent case. Figure 2(a) shows the maximal growth rate for fixed $\gamma, \mathrm{U}_{W}$ and $2 \pi n_{\xi} \beta_{0} / L_{\xi}$. For $\left|2 \pi n_{\xi} \beta_{0} / L_{\xi}\right|$ sufficiently small, the maximal growth rate monotonically decreases as $L_{1} / L_{j}$ decreases, similar to the $\xi$-independent case in Figure 1. This is to be expected, since $2 \pi n_{\xi} \beta_{0} / L_{\xi}=0$ corresponds to the $\xi$-independent case. On the other hand, for larger $\left|2 \pi n_{\xi} \beta_{0} / L_{\xi}\right|$, the plots of the maximal growth rate show that these have maxima at $\mathrm{L}_{1} / \mathrm{L}_{\mathrm{J}} \sim 10^{1 / 2}$. The position and magnitude of these maxima both decrease as $\left|2 \pi n_{\xi} \beta_{0} / \mathrm{L}_{\xi}\right|$ becomes larger, but the maximal growth rate for larger $\left|2 \pi n_{\xi} \beta_{0} / L_{\xi}\right|$ does not exceed that for smaller $\left|2 \pi n_{\xi} \beta_{0} / L_{\xi}\right|$ even at $\mathrm{L}_{1} / \mathrm{L}_{\mathrm{J}} \sim 10^{1 / 2}$. Hence, the maximal growth rate for a fixed $\mathrm{L}_{1} / \mathrm{L}_{\mathrm{J}}$ monotonically decrease as $\left|2 \pi n_{\xi} \beta_{0} / L_{\xi}\right|$ increases, and this will be confirmed later. These maxima appear for different choices of $U_{W}$ and $\gamma$ (not shown). The mechanism of the appearance is not yet clear, and the structure of the eigenfunctions shows no apparent change from those of large or small $\left|2 \pi n_{\xi} \beta_{0} / L_{\xi}\right|$. Now, concentrating on the region where $\mathrm{L}_{1} / \mathrm{L}_{\mathrm{J}}$ is small, all the maximal growth rates appear to asymptote to zero. However, for the same reason as stated for the $\xi$-independent case in Subsection 3.1, they do not become zero but remain positive.

The plot of maximal growth rate for fixed $\gamma, \mathrm{U}_{W}$ and $\mathrm{L}_{1} / \mathrm{L}_{\mathrm{J}}$ is shown in Figure 2(b). ${ }^{4}$ These monotonically decrease as $\left|2 \pi n_{\xi} \beta_{0} / L_{\xi}\right|$ increases, and take the maximum value at $2 \pi n_{\xi} \beta_{0}=0$, whether or not they have a lo-

${ }^{4}$ We could not obtain the eigenvalue for large $\left|2 \pi n_{\xi} \beta_{0} / L_{\xi}\right|$ because continuous modes appear and the numerical calculations no longer converge in this limit. 
Figure 2: (a) Maximal growth rate for $\gamma=1.0, \mathrm{U}_{W}=-1.0,2 \pi n_{\xi} \beta_{0} / \mathrm{L}_{\xi}=$ $1.0 \times 10^{-4}$ (red), $5.0 \times 10^{-4}$ (orange), $1.0^{-3}$ (blue), $1.1 \times 10^{-3}$ (green), and $1.2 \times 10^{-3}$ (light blue). (b) Maximal growth rate (red crosses) for $\gamma=1.0$, $\mathrm{U}_{W}=-1.0, \mathrm{~L}_{1} / \mathrm{L}_{\mathrm{J}}=1.0$ (left) and 3.0(right).
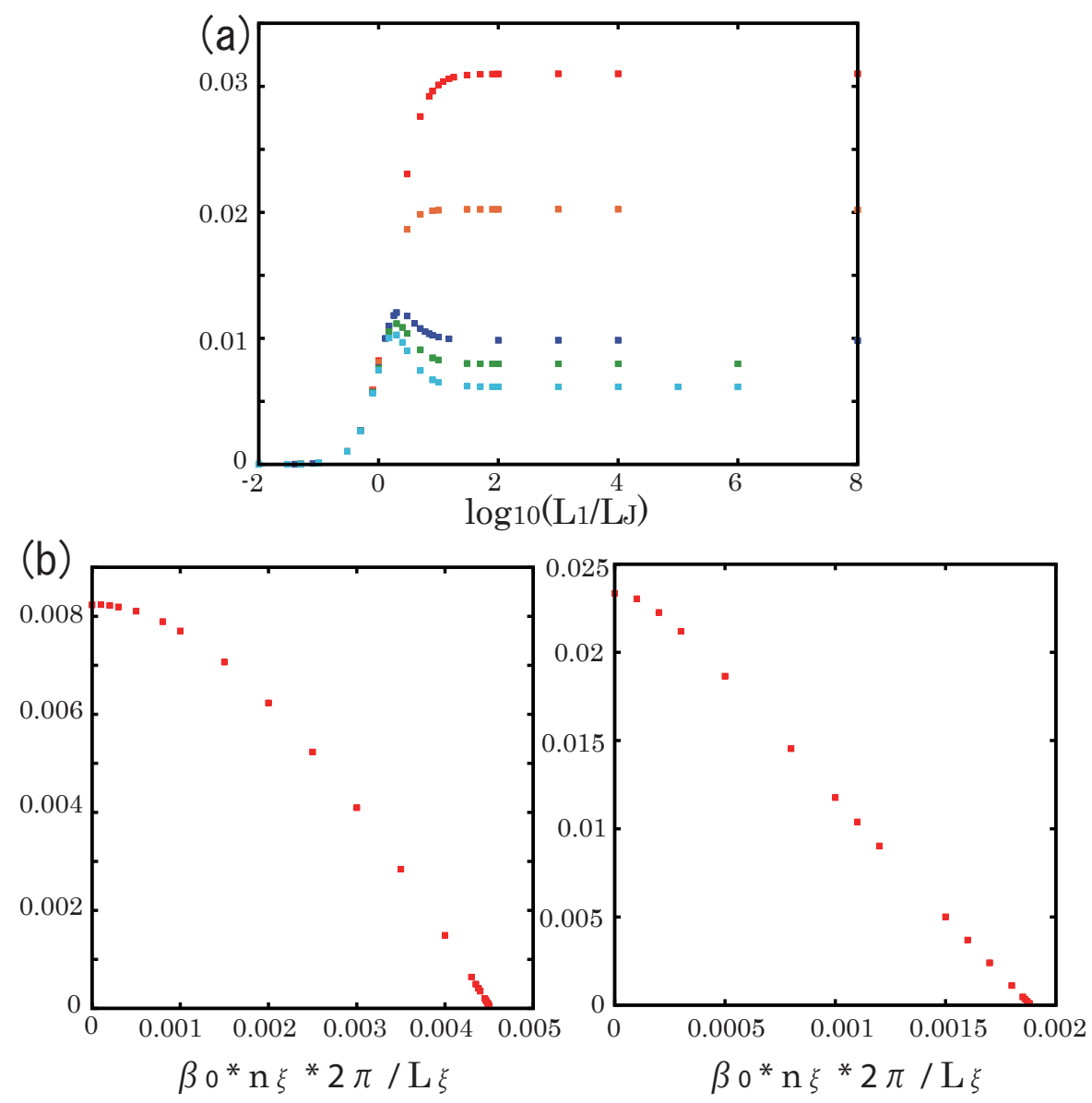
cal maximum in Figure 2(a). This confirms that the maximal growth rate at larger $\left|2 \pi n_{\xi} \beta_{0} / L_{\xi}\right|$ does not exceed that for smaller $\left|2 \pi n_{\xi} \beta_{0} / L_{\xi}\right|$ in Figure 2(a).

Furthermore, Figure 2 suggests that all the zonal jet solutions $\mathrm{U}_{0}$ are linearly unstable, and specifically, the $\xi$-independent mode, i.e., the mode independent of longitudinal direction, is the most unstable mode. This is even though the amplitude equation on the zonal flows (7) has both $\eta$ - and $\xi$-dependence. The instability is expected to bring about the deformation of the steady isolated zonal jet $\mathrm{U}_{0}$ and cause its disappearance.

\section{Conclusions}

In this paper we have performed an asymptotic analysis in an equivalentbarotropic quasi-geostrophic system, extending Manfroi and Young's analysis [3]. We then studied the linear stability of steady isolated zonal jet solutions $\mathrm{U}_{0}$ when it is governed by an amplitude equation that is independent of and dependent on the longitudinal variable $\xi$. It was found that in both cases all the zonal jet flows $U_{0}$ under the influence of zonal flow-nonzonal background flow interaction are linearly unstable. Consequently, the zonal jet solutions are expected to deform in time. For the case where the amplitude equation of the zonal flows has longitudinal dependence, it was also found that the mode independent of longitudinal direction, i.e., the zonal mode, is most unstable among all the two-dimensional modes. We are particularly interested in long-time behaviour and the final state of the steady isolated zonal jets $\mathrm{U}_{0}$ in these systems, especially when $\mathrm{U}_{0}$ is allowed to have non-zonal variation. This problem is now under investigation.

Acknowledgements Some of the data analysis and visualisations in this paper were done with the software packages ISPACK, gt4f90io, spmodel [5] and the software products of the GFD Dennou Ruby project. The numerical 
calculations were performed using the computer systems of the Institute for Information Management and Communication, Kyoto University and of the Research Institute for Mathematical Sciences, Kyoto University.

\section{References}

[1] K. Obuse, S. Takehiro and M. Yamada, Long-time asymptotic states of forced two-dimensional barotropic incompressible flows on a rotating sphere. Phys. Fluids 22:156601, 2010. doi:10.1063/1.3407652 C176

[2] R. K. Scott and L. M. Polvani, Forced-dissipative shallow-water turbulence on the sphere and the atmospheric circulation of the giant planets. J. Atmos. Sci. 64:3158-3176, 2007. doi:10.1175/JAS4003.1 C176

[3] A. J. Manfroi and W. R. Young, Slow evolution of zonal jets on the beta plane. J. Atmos. Sci. 56:784-800, 1999. doi:10.1175/1520-0469(1999)056<0784:SEOZJO>2.0.CO;2 C176, C177, C178, C179, C186

[4] K. Obuse, S. Takehiro and M. Yamada, Linear stability of steady zonal jet flows induced by a small-scale forcing on a $\beta$ plane. Physica D 240:1825-1834, 2011. doi:10.1016/j.physd.2011.08.009 C176, C179, C181, C182, C183, C184

[5] S. Takehiro, M. Odaka, K. Ishioka, M. Ishiwatari, Y.-Y. Hayashi and SPMODEL Development Group, A series of hierarchical spectral models for geophysical fluid dynamics. Nagare Multimedia, 2006. http://www. nagare.or.jp/mm/2006/spmodel/ C186 


\section{Author addresses}

1. K. Obuse, Graduate School of Environmental and Life Science, Okayama University, Okayama, 700-8530, JAPAN. mailto:obuse@okayama-u.ac.jp

2. S. Takehiro, Research Institute for Mathematical Sciences, Kyoto University, Kyoto, 606-8502, JAPAN.

3. M. Yamada, Research Institute for Mathematical Sciences, Kyoto University, Kyoto, 606-8502, JAPAN. 